\title{
Changing by light - how the lighting of intelligent spaces can influence the urban image
}

\author{
K. Życzkowska \\ Gdansk University of Technology, Poland
}

\begin{abstract}
In the article light will be presented as a tool in the process of transforming urban space. The reason for this use of light is the commercial aspect of space that forces it to be still competitive and profitable. Such a need gives rise to many innovative solutions in the city space, which allow the transmitting of information in order to attract human attention, and sometimes even make it possible to involve the user in the interaction with the space. These kinds of efforts take advantage of the intelligence of space, which makes architecture integrated with information technology and, as a result, uses lighting technology in the process of creating changing images of spaces. The article will present the influence of lighting technology on commercial space and will show solutions that increase the attractiveness of space. They will be presented depending on localization (from significant façades to less known, hidden places) and duration in the space (from fixed elements to temporary events). Moreover, the relation between the user and the space will be taken into account.
\end{abstract}

Keywords: light in architecture, light in commercial spaces, intelligent spaces, media façades, light installations.

\section{Introduction}

Light is not only indispensable for our everyday tasks, but can also be used to create the atmosphere and to build a sense of safety in the space. What is more, light allows us to change the appearance of the architectural surroundings and make it more dynamic and interesting for the users of the space. While talking about light as a tool for modifying the urban space we should take into 
consideration the commercial aspect of a city, which is very often the reason for the new ways of using light - mainly as a form of promotion of a given place.

The commercial space must bring profits, but it should also be useful and follow the needs of its users. That is why it has to develop, look for new forms of publicity and attract new users through the innovations increasing the attractiveness of the space. It is a very complex issue, but one of the factors that have influence on the quality of commercial space is the intelligence of that space.

The intelligence of space means ability of space to react to the changes in its environment. The intelligence of space can be understood at the level of administration of the intelligent building (optimization of a building's life, safety and security), however, it will not be the subject of this article. The definition of intelligence for the purpose of this article is the synergy of architecture, information technology (IT) and art, which allow one to use lighting technology to change the images of the urban space in real time. It is described at the level of communication with the space - from visible transmissions of information (commercial and non-commercial content) and the influence on human perception, to the possibility of interaction between the user and the space.

\section{From billboards to media façades}

It is important to explain, that if lighting technology, even integrated with IT, does not follow the architectural functionality and structure, we cannot talk about intelligence of the space.

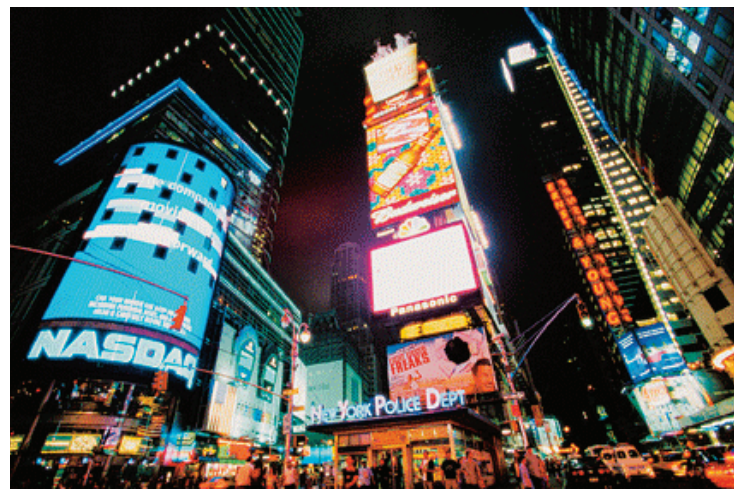

Figure 1: Times square; from the left: NASDAQ MarketSite and One Times Square [1].

To make it clear, let us start with Times Square in New York - a space filled with plenty of LED-billboards, which almost cry to attract users' attention. It is hard to believe that the One Times Square - a 110.6m high skyscraper which used to be the headquarters of The New York Times - is nowadays only an empty frame to hold dozens of large advertisements since, apart from retails situated in the lowest part of the building, there are no tenants inside (Fig. 1). 
Moreover, such a use of the building brings bigger profits than it would generate by being completely rented (what would be connected with a significant renovation). On the one hand, due to its revenue, the building fulfils the requirements for the commercial spaces, but of course we cannot treat this space as an intelligent space. It is not the desired role of lighting technology to cover the architectural structure by LED high definition screens and to take its function away.

First video cladding that solved the problem of illumination of the building's interior by natural light appeared in 1996 on the façade of NASDAQ MarketSite in Times Square. However, it is not a very sophisticated solution, because this 10 -story and $1000 \mathrm{~m}^{2}$ video wall in the bottom corner of Condé Nast Building was simply pierced by the window slots (Fig. 1).

However, there are more advanced solutions that can bring façade of a building into the new level of intelligent billboard. As an example the headquarters of T-Mobile in Bonn can be given. Its front of $300 \mathrm{~m}^{2}$ was transformed in 2003 into a huge LED video screen, which on the one hand transmits the changing images on its surface and at the same time is transparent during the day and allows natural light to access the inside of the building (Fig. 2). Such functionality is possible thanks to the technology of mediatecture designed by ag4 - an architectural and media communications company (Cologne, Germany), in collaboration with GKD (Düren, Germany) - a manufacturer of metal woven building mesh. It is an unobtrusive wire mesh where rows of steel sleeves hold the transparent aluminium profiles covered with a waterproof resin and filled with RGB LEDs.
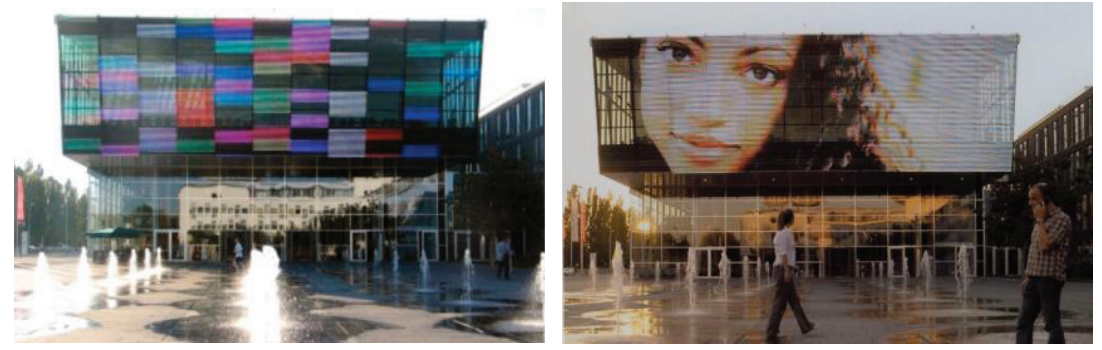

Figure 2: $\quad$ T-Mobile, Bonn, from left: [2, 3].

The T-Mobile façade is a first realization where this solution was applied, however it can be adjusted to different sizes of façades by regulation of distance between the rows of the mesh. Mediamesh allows transmitting varied content as text, pictures and video images, and with the use of suitable software also enables the presentation of live video. Another important issue is the fact that the projections are obtained in low resolution preventing the video screen from prevailing in its surroundings. Moreover, T-Mobile's façade is a medium that is not only reserved to advertising signs but also to art and culture projections.

This approach to share the urban screens between commercial and noncommercial transmissions is becoming more and more popular, so as the 
embodying information technology into architectural structure. The next example that confirm this thesis is the spectacular façade of Grand Indonesia Tower in Jakarta (2009), designed Darryl Yamamoto, AIA, director of Austin Veum Robbins Partners (AVRP) and Mixed Use Studio (both of Los Angeles) (Fig. 3).

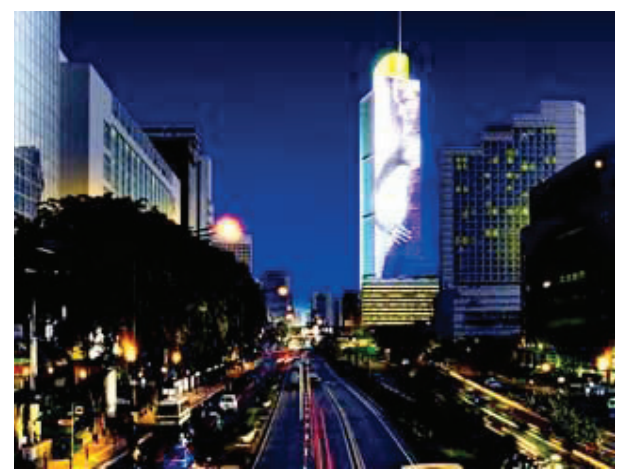

Figure 3: $\quad$ Grand Indonesia Tower, Jakarta [4].

Here about $60,000 \mathrm{ft}^{2}$ (more than $5,570 \mathrm{~m}^{2}$ ) of LED arrays are integrated with entire front curtain wall in several types of formations. The content of this media façade consists of one half of profitable publicity and the second of the artistic projections and public service announcements.

Worthy of note is also another commercial building which not only is covered by media façade all around its external surface, but is also completely free of commercial content. It is the Galleria Centercity in Cheonan, in South Korea (2009), created by UN Studio (The Netherlands) with GANSAM (Seoul) and companies: AG Licht (Bonn, Germany) and Licht Kunst Lich, responsible for the realization of lighting technology (Fig. 4). Such an approach to media façade is a result of actual behavioural tendencies concerning the commercial space. It is not just the shopping centre, but also the place of meeting and spending time, therefore the social aspect of such a space is very important. That is why the

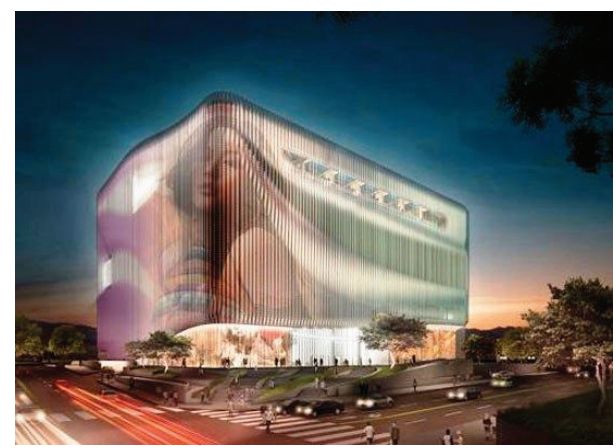

Figure 4: Galleria Centercity department store, Cheonan [5]. 
light in the intelligent, commercial space is used not only as a tool to transmit concrete information, but also to create more abstract impressions - to change the surroundings into more interesting and more attractive place in order to make people more familiar with the space and encourage them to stay in it and use it.

Another media façade that does not transmit commercial essence is ILUMA building in Singapore (2009) - a shopping complex designed by Realites United and WOHA (Fig. 5). An interesting feature of this structure is the fact that it integrates the elements of a façade with lighting technology through the system called Crystal Mesh. It consists of spatial elements of characteristic crystal shape, which hide 1 to 7 fluorescent, energy-efficient bulbs each and are covered by layer of polycarbonate. The system allows us to obtain the impressions of depth of graphical images.

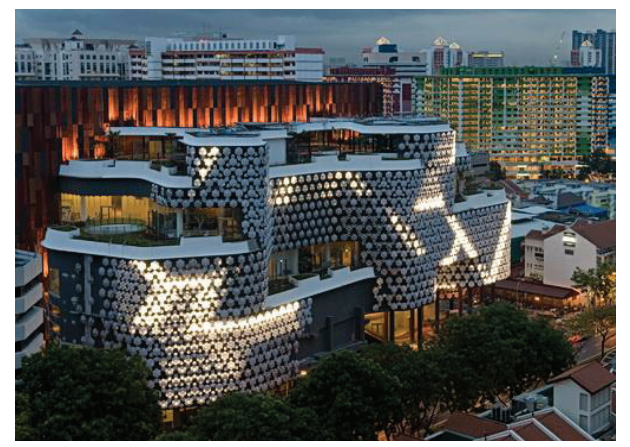

Figure 5: $\quad$ ILUMA building, Singapore [6].

\section{Lighting installations as a form of promotion of the space}

The promotion of a space does not have to consist of the direct commercial content, which is confirmed by the approach of contemporary media façades. To prove this thesis the competition of European Capital of Culture can be also mentioned. Taking part in this action is connected with efforts taken up by the cities to make their images more attractive, to become renowned on an international scale, and as a result bring cultural, social and economic profits for the city. One of the means on the way to obtaining this title can be organization of festivals of light. Such an action took place twice in Gdansk - Polish candidate in this competition. The event is called Narracje (Narrations) and in 2010 in Lost \& Found edition it involved 15 artists who changed the main town's space by visual art (Fig. 6).

In the projects carried during the event the artists were trying to influence human perception using abstract patterns to transform in real time the façades of historical buildings - for example: St Jean Church by Danuta Kiewłen (Poland) or the building on the Granary Island by Ghiju Diaz de Leon (Mexico). Other artists, from Projektil (Switzerland), proposed an installation that could even be modified by the users. On the façade of one of the old tenement houses next to 

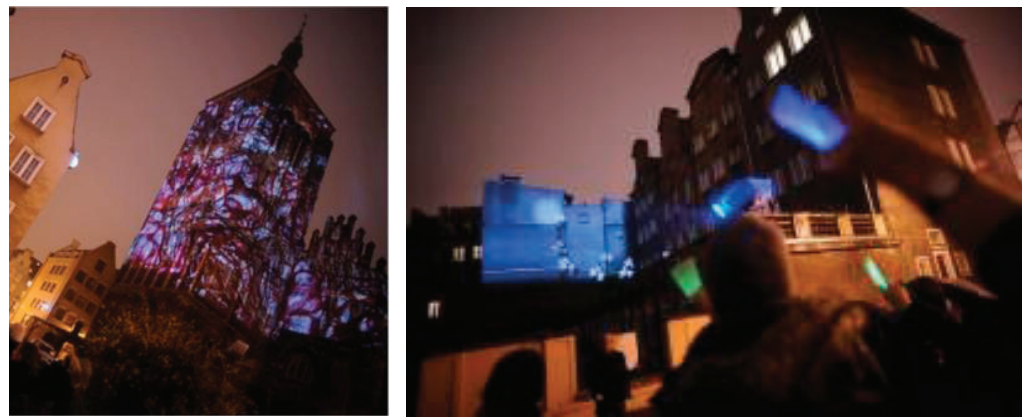

Figure 6: Narracje, Lost \& Found, Gdansk, from the left: St Jean Church and Kozia Street [8].

Kozia Street the artists organized a virtual garden with the use of video mapping technology. Using special light indicators or mobile phone screens in suitable colour (green, blue or red) the viewers could provoke the sprouting of plants, appearing of flowers or destruction of visual crops to start the game one more time [7].

It is important to stress that the solutions that involve users in creation of the change by interaction with the space do not have to be organized as temporary events but can also constitute a part of fixed architectural structure. Such an example is EnterActive in Met Lofts (Los Angeles), created in 2006by Electroland [9].

Here the behaviour of people on the light carpet in the lobby of this apartment building is transmitted into the light pattern on its façade. It is possible because the interior floor divided into grid of electronic tiles is joined by a computer program with a similar pattern of illuminated square elements on the façade (Fig. 7). There are 176 tiles on the floor and each of them consists of a layer of fritted glass and plastic that holds 96 red LEDs and is also equipped with 4 sensors of compression and a microcomputer from below. It allows them to illuminate the tiles when somebody steps on them and send the signal through the master computer to the façade in order to illuminate it according to the movement in the lobby.
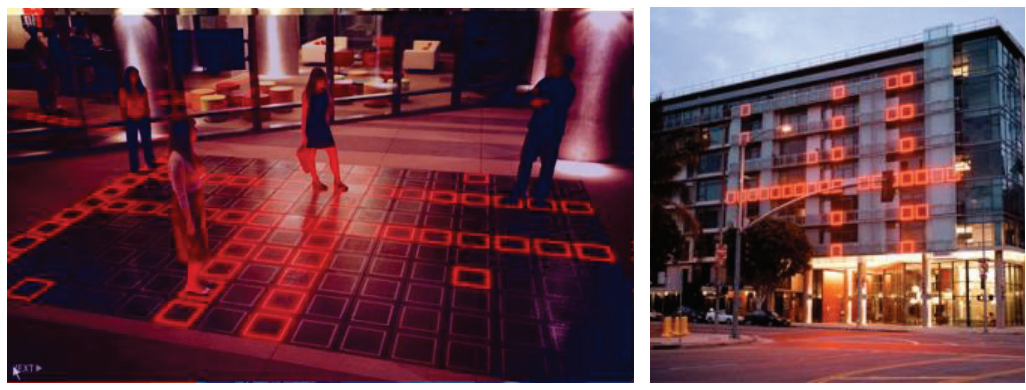

Figure 7: $\quad$ EnterActive - intelligent floor and façade [10]. 
So as the image of walls or floor of the architectural surroundings can be changing by the intelligence of space, the similar effect can be obtain in reference to the ceiling. This kind of solution was applied to the stunning project presented at the CeBIT 2005 trade show. It was designed by Munich Studios' KMS Team and Schmidhuber + Partner for Mobile telephone provider O2 Germany. The installation called the Media Cloud consisted of over 28,000 points of light, which, while being still in motion, could transmit changing images above the heads of spectators (Fig. 8). This enormous, low-resolution display of $1,123 \mathrm{~m}^{2}$ consisted of satin-frosted $5 \mathrm{~cm}$ diameter tubes of different length which were suspended from the ceiling at intervals of $20 \mathrm{~cm}$, and where Versa Pixels (product line consisting of RGB LEDs designed by Element Labs) were placed. Obtained resolution constituted of $243 \times 112$ pixels, what gave the brightness at a level of about 1,100 nits (candela per $\mathrm{m}^{2}$ ).

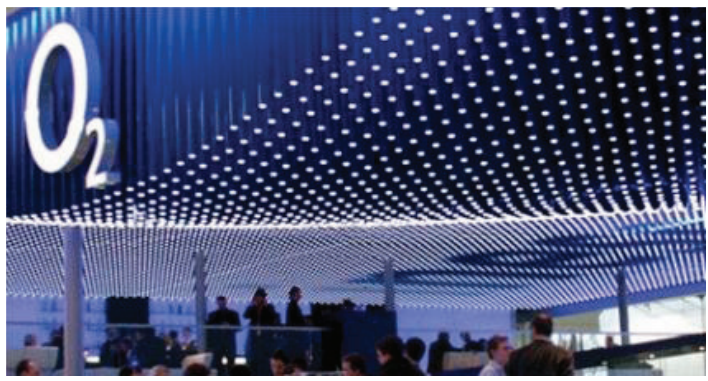

Figure 8: $\quad$ Media Cloud - intelligent ceiling [11].
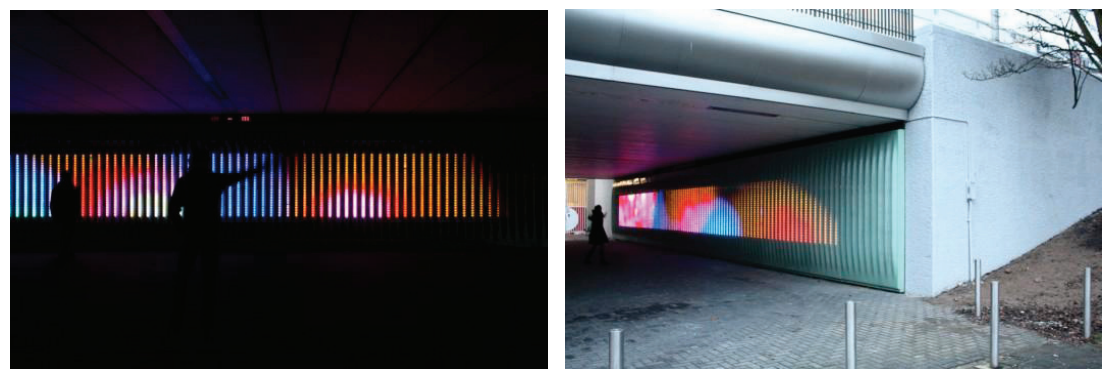

Figure 9: Moodwall, Amsterdam [12].

It is time to add that the idea of lighting installations does not have to concern only the most significant and visible parts of the space of our cities. Such solutions can be also implemented in the zones which are rather hidden in the urban space, but are also used and should guarantee the sense of safety and bring positive connotations. An example which uses a light installation to influence the impressions in urban space is The Moodwall created by Urban Alliance - an interactive light installation situated in a pedestrian tunnel of Amsterdam (Fig. 9). It is a 24 meter long wall embodied with about 2,500 LEDs which 
interacts with passers-by who, by their movement, provoke the changes in colourful projections appearing on its surface. This game with the light makes the space of this passage more interesting, brighter and safer at the same time.

The intelligence of a space was also applied to another pedestrian subway. This time it was in 2009 in Sandnes, in Norway, where Snøhetta Architects used Dobpler Interactive Led System Lighting - the work of Skjelvik Designv - in order to realize the project called Strømmer [13]. The interactive wall of this passage allows luminous shadows to be represented in low resolutions of pedestrians passing through the subway.

Another example of the intelligent space located underground is the Sunderland station in Sunderland, in United Kingdom (Fig. 10). In this case Sadler Brown Architecture in cooperation with Arup and artist Jason Bruges made the station more interesting, illuminated and dynamic by incorporating a $144 \mathrm{~m}$ long wall of glass blocks which are in fact the pixels of low resolution video screen.

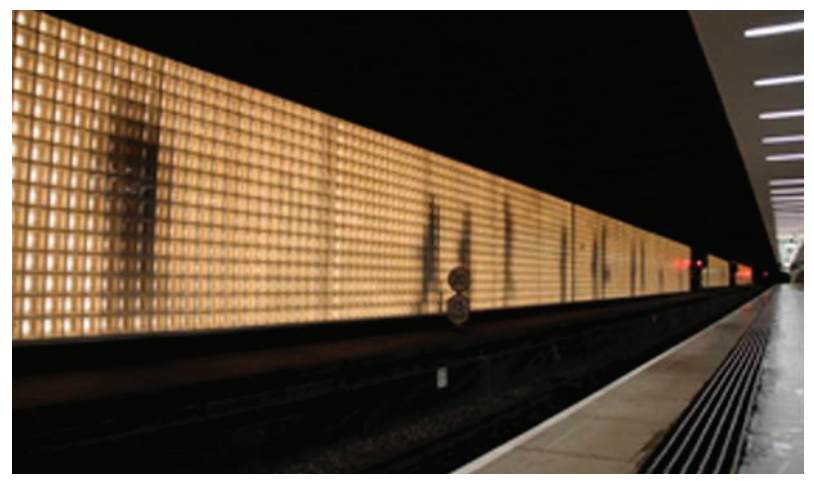

Figure 10: $\quad$ Station in Sunderland [14].

The bright surface transmits darker shadows of virtual travellers. The projection is cleared every time the train comes to the platform giving the illusion that these unreal passengers can board the train and leave the platform. This kind of solution has a positive influence on the atmosphere of the station. It consist of eW Flex SLX type lighting fittings, LED technology of lighting and LOSH type wiring system [15].

While talking about spaces connected with traveling, the realization called Travelling Sand from Apeldoorn in The Netherlands from 2008 is also worth mention [16]. The installation was designed by Giny Vos in collaboration with $3 \mathrm{D}$ animator Bram Verhavert. It is a $100 \mathrm{~m}$ long intelligent glass wall covering the entrances to the platforms of a railway station that consists of about 1,3 million LED. It creates a landscape of dunes modified by virtual wind and makes the surroundings of the railway station an unusual space. 


\section{Conclusions}

Summing up, architecture integrated with information technology and visual art treats light as a tool to transform the commercial space. The intelligence of space has a huge influence on the appearance of the city and also on people's behaviour. A process of changing the space agreeable with this approach can be realized by fixed elements in the space (like media façades, elements of interiors or underground passages) or temporary events, using lighting installations for some occasional purposes. Intelligence of the space can appear in the significant, central commercial space and in more hidden urban spaces. The possibilities of using light are enormous, however it is very important to look for suitable solutions for each kind of space, remembering also about the need to find a balance between the technology, content and human acceptation of space. Using light in commercial space does not have to be limited only to billboards that attack the users by aggressive, commercial transmissions. There is also another approach which involves introducing lighting installations into more abstract and artistic projections for promotion purposes. This form of dynamisation and personalization of space can attract new users and make them feel better in such a space. Not only is it a result of perception of the changing images of the space but it happens also due to the possibility of interaction with the space which makes users able to provoke changes in their surroundings.

\section{References}

[1] http://www.themeetingmagazines.com/index/Default.aspx?tabid=366

[2] http://www.mimoa.eu/projects/Germany/Bonn/T-Mobile\%20Headquarters

[3] http://nait5.com/2008/06/03/t-mobile-headquarters/

[4] http://www.screens.ru/en/2006/7.html

[5] http://www.sztuka-architektury.pl/index.php?ID_PAGE=21965

[6] http://www.mediaarchitecture.org/uec-iluma-singapore-2/

[7] Zięba K., Gdańsk błyszczy, Architektura, 02/2011, pp. 28-29, 2011.

[8] http://kultura.trojmiasto.pl/Narracje-czyli-rozswietlony-nocny-Gdanskn43431.html

[9] Hart H., I am a Camera: Electroland, AD (Architecture \& Design) 4dsocial Interactive Design Environments, vol 77, n 4, 07-08/2007, pp. 88-97, 2007.

[10] http://archimedespool.wordpress.com/2007/08/29/enteractive-contentdigital_communications-lighting/

[11] https://archimedespool.wordpress.com/2007/09/18/o2-on-cebit-2005/

[12] http://www.archdaily.com/23239/moodwall-studio-klink-and-urbanalliance/

[13] http://dobpler.com/

[14] http://www.sadlerbrown.co.uk/news.aspx?item=25

[15] Slavid R., Stacja Sunderland, Sunderland, Wielka Brytania, Peron duchów, Luminous, 06/2011, pp. 5-7, 2011.

[16] Ricchi D., 2008, Giny Vos Travelling Sand, MATERIA, n 58, pp.112-119, 2008. 